\title{
Caracterización dinámica vibratoria experimental de compuestos reforzados con fibra natural de fique
}

\author{
Experimental dynamic characterization of composites \\ reinforced with natural fiber of fique
}

\author{
Sergio Andrés Gómez Suárez ${ }^{1 *} \quad$ Bladimir Azdrubal Ramón Valencia ${ }^{2} \quad$ Alfonso Santos Jaimes ${ }^{1}$ \\ Recibido 12 de julio de 2018, Aceptado 31 de enero de 2019 \\ Received: July 12, 2018 Accepted: January 31, 2019
}

\begin{abstract}
RESUMEN
Los materiales biocompuestos reforzados con fibras naturales juegan un papel importante en estos días para el desarrollo de nuevos productos debido a que ofrecen buenas características mecánicas, reduciendo el impacto ecológico negativo a la hora de su desecho. Su uso en aplicaciones dinámicas aun es limitado, ya que no hay suficientes estudios donde se caractericen sus comportamientos en estos ambientes. Usualmente se aplica el ensayo dinámico mecánico DMA para conocer las propiedades térmicas - mecánicas de este tipo de materiales sin embargo esta técnica no brinda información de los parámetros modales como lo son las diversas frecuencias de vibración en la que el mismo entraría en resonancia. Para obtener estas propiedades se debe realizar un análisis modal. Por tal motivo en este trabajo se realizó una caracterización dinámica vibratoria por medio de análisis modal experimental a biocompuestos reforzados con fibras de fique evaluando el impacto en los parámetros modales debido al contenido de la fibra natural lo cual permitió evidenciar cómo se comporta los biocompuestos de fique a cargas vibratorias, ya que no se cuenta con evidencia de estudios anteriores de esta clase a biocompuestos con este tipo de fibra natural, y en que posibles aplicaciones podrían utilizarse. Los biocompuestos fueron elaborados con diferentes capas de fibra natural de fique en distribución aleatoria y una resina poliéster mediante la técnica de manufactura de infusión en vacío. El análisis modal experimental se realizó obteniendo la medición de la respuesta en frecuencia (FRF) según Norma ISO 7626-2 excitación con shaker. Los resultados obtenidos evidencian un incremento en la frecuencia natural de los biocompuestos a medida que se le incorpora más cantidad de fibra natural presentando adicionalmente un alto amortiguamiento y modos flectores de vibración, así como una posible aplicación como aislantes de vibración y sonido.
\end{abstract}

Palabras clave: Análisis modal, biocompuesto, fique, parámetros modales.

\section{ABSTRACT}

The biocomposite materials reinforced with natural fibers play an important role these days for the development of new products. They offer good mechanical characteristics, reducing the negative ecological impact at the time of their disposal. Its use in dynamic applications is still limited since there are not enough studies where its behavior is characterized in these environments. DMA mechanical dynamic test is usually applied to know thermal-mechanical properties of this type of materials. However this technique does not provide information on the modal parameters of the materials, as are the different vibration

\footnotetext{
1 Universidad Pontificia Bolivariana. Facultad de Ingeniería Mecánica. Bucaramanga, Colombia. E-mail: sergiogomezs@upb.edu.co; alfonso.santos@upb.edu.co

2 Universidad de Pamplona. Departamento de Ingeniería Mecánica. Pamplona, Colombia. E-mail: hbladimir@unipamplona.edu.co

* Autor de correspondencia: sergiogomezs@upb.edu.co
} 
frequencies in which it would come into resonance. To obtain these properties, a modal analysis must be performed. Therefore, in this work a dynamic vibratory characterization was made through experimental modal analysis to biocomposites reinforced with fique fibers, evaluating the impact in the modal parameters due to the natural fiber content, which allowed recognizing how the biocomposites behave due to vibratory loads since there is no evidence of previous studies of this kind to biocomposites with this type of natural fiber and in which possible applications could be used. The biocomposites were made with different layers of natural fiber from fique in random distribution and polyester resin using the technique of vacuum infusion manufacturing. The experimental modal analysis was performed obtaining the frequency response function (FRF) according to ISO 7626-2 excitation with a shaker. The results obtained show an increase in the natural frequency of the biocomposites as more natural fiber is incorporated, presenting a high damping and bending modes of vibration as well as a possible application as vibration and sound insulators.

Keywords: Modal analysis, biocomposites, fique, modal analysis.

\section{INTRODUCCIÓN}

La planta de fique se usa principalmente para la elaboración de cuerdas y empaques en países americanos del trópico como Colombia, Brasil, Ecuador, Costa rica y las Antillas aportando ingresos económicos importantes a poblaciones agrícolas que viven de su cultivo. Sin embargo, debido a la superproducción de plásticos y a la entrada de las fibras sintéticas, este sector ha entrado en crisis [1]. Esta situación ha obligado a los productores e investigadores en fibras naturales a buscar otros usos para las fibras de fique, que implique aplicaciones industriales como refuerzo de materiales compuestos de matrices poliméricas [2-3].

Un material compuesto es la unión de dos o más materiales para adquirir propiedades que no son posibles de obtener de manera independiente; los mismos se conforman por una matriz y por un refuerzo [4], utilizándose en diversas aplicaciones de sectores industriales como el aeronáutico, naval, construcción , automotriz, eléctrico, entre otros [5-6].

El uso del fique como refuerzo de materiales biocompuestos, ha brindado ventajas sobre los polímeros como: la disponibilidad, renovación, baja densidad, resistencia a corrosión, bajo costo y biodegradabilidad [7-8]. No obstante, su carácter hidrófilo no permite formar adhesiones fuertes con la matriz polimérica de comportamiento hidrofóbico, afectando las propiedades finales del material compuesto [9].

Diversas caracterizaciones se han aplicado a los biocompuestos reforzados con fibras de fique para conocer sus propiedades más representativas como se menciona a continuación.

Morfológicamente, Hidalgo et al. [10] caracterizaron un biocompuesto PEBD-Al/ fique utilizando microscopía electrónica de barrido (SEM). Las imágenes de SEM revelaron una pobre interface entre la fibra y la matriz. En otro estudio, Hidalgo et al. [11] reportaron el mismo efecto entre las fases de un biocompuesto de LDPE-Al/fique.

Mina et al. [12] y Navia et al. [13] caracterizaron mecánicamente un biocompuesto de almidón termoplástico/fique y un biocompuesto harina de yuca/ fique respectivamente, concluyendo que el esfuerzo de tensión y el módulo de elasticidad aumentan con mayor cantidad de fibra natural utilizada. Así mismo Arjona et al. [14] caracterizando biocompuesto $\mathrm{PEAD} /$ fique y Gómez et al. [15] un biocompuesto epoxy/fique, demostraron que el esfuerzo y el módulo de flexión también aumentan a mayor contenido de fibra natural.

Ensayos dinámicos-mecánicos DMA, de materiales compuestos reforzado con fibras de fique y matriz polimérica han sido reportados en la literatura, brindando información de las propiedades dinámicas (visco-elásticas) como el módulo de almacenamiento, tan delta y el módulo de pérdida [16-18]. Sin embargo, este tipo de ensayo no brinda información suficiente relacionada con otros fenómenos dinámicos relevantes como las frecuencias de vibración en las que los materiales entrarían en resonancia.

El análisis modal es la técnica que determina el comportamiento vibratorio de los materiales, que 
siendo aplicada por medio de métodos experimentales que miden la respuesta en frecuencia (FRF), permite obtener los parámetros modales de un cuerpo como la frecuencia natural (frecuencia a la que un sistema mecánico vibra en condición libre y sin excitación), el amortiguamiento (capacidad del cuerpo para disipar energía) y la forma modal (referente a la deformación periódica consecuente en la pieza producto de la vibración) [19].

Varios son autores que han realizado estudios en materiales compuestos reforzados con fibras mediante el uso de esta técnica (FRF), especialmente con fibras de carbón y vidrio [20-23]. Sin embargo, no hay suficiente información sobre materiales compuestos reforzados con fibras naturales [24]. Por tal razón, el presente trabajo tiene como objeto caracterizar dinámicamente biocompuestos reforzados con fibras naturales de fique, haciendo uso del análisis modal experimental para evaluar el impacto de los parámetros frecuencia natural, amortiguamiento y forma modal, con respecto a la variación del número de capas de fibras en el compuesto.

\section{MÉTODOS}

A continuación, se describen los diferentes materiales y procesos de manufactura utilizados para la fabricación de los biocompuestos, igualmente la metodología que se utilizó para el análisis modal experimental.

\section{MATERIALES}

Fibras naturales de fique en forma aleatoria, fueron utilizadas como refuerzo de los materiales compuestos suministradas por la fundación San Lorenzo de Barichara, Colombia. En la Figura 1, se observa su topografía.

Las fibras de fique recibieron tratamiento térmico por 12 horas a una temperatura de $70^{\circ} \mathrm{C}$ con el objetivo de eliminar la humedad. Después del proceso térmico fueron maceradas para la obtención de fibras cortas. Las fibras obtenidas se clasificaron utilizando un tamiz artesanal de madera. El arreglo aleatorio se prenso en máquina industrial por ocho días obteniéndose como resultado hojas con un espesor de 0,31 $\pm 0,12 \mathrm{~mm}$.

Las fibras cortas de fique utilizadas presentaron una longitud promedio de $1,76 \pm 0,53 \mathrm{~mm}$ y un diámetro de $0,0253 \pm 0,0033 \mathrm{~mm}$, como se evidenció en la realización de la medición por medio de microscopia electrónica de barrido SEM en un estudio anterior realizado por los autores [25].
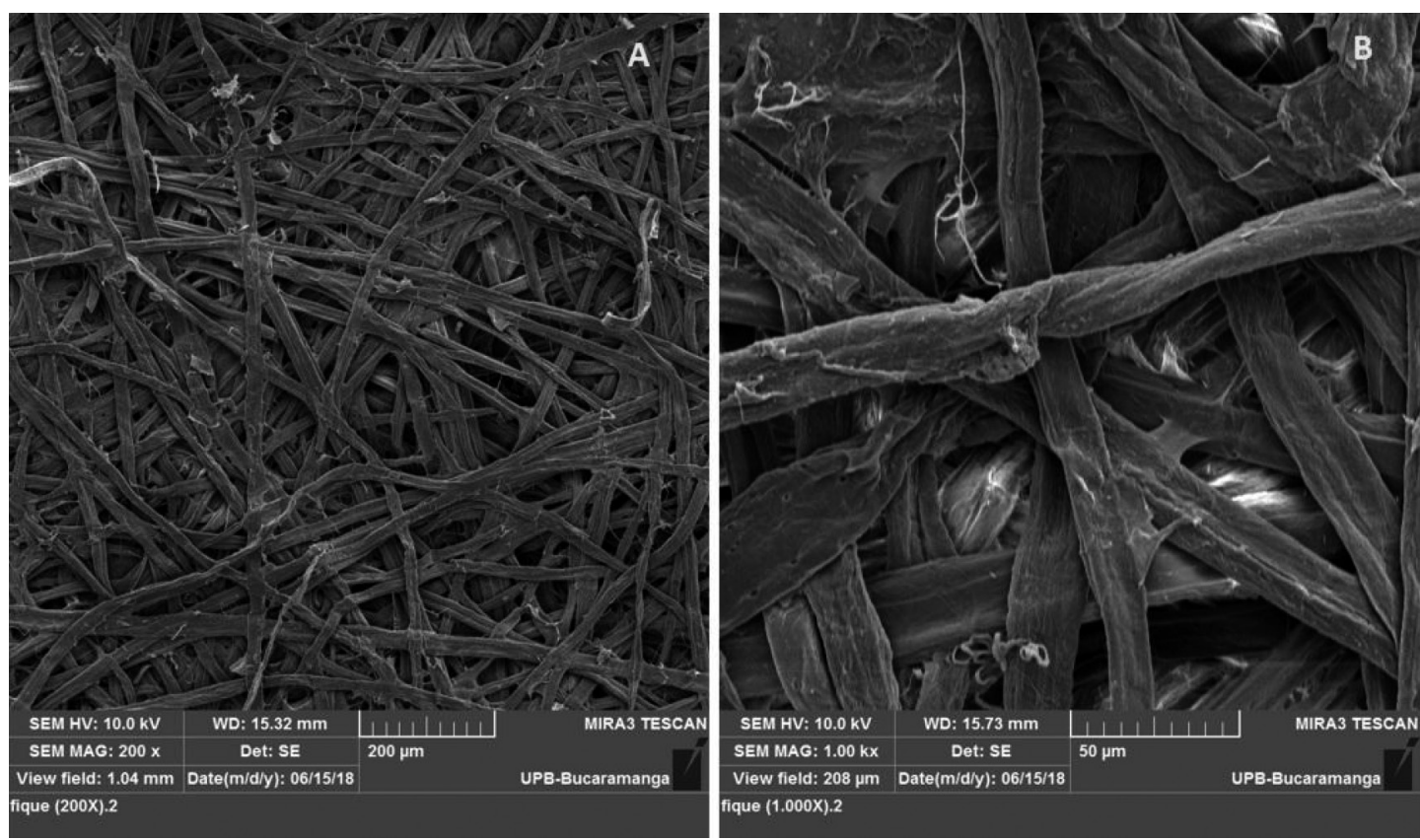

Figura 1. Micrografías SEM del fique organización aleatoria A) escala 200X B) escala 1000X. 
Para los compuestos con diferentes capas de fique se utilizó como matriz una resina polimérica de poliéster referencia 856 suministrada por la empresa Ingequímicas de Bucaramanga Santander, Colombia. La relación en peso resina/catalizador fue de 100:2.

En la Tabla 1 se relacionan algunas propiedades, suministradas por el proveedor, del poliéster utilizado para la elaboración del compuesto.

Tabla 1. Propiedades mecánicas de la resina de Poliester utilizada.

\begin{tabular}{|l|c|}
\hline \multicolumn{1}{|c|}{ Propiedad } & Magnitud \\
\hline Esfuerzo Tensión (MPa) & 11 \\
\hline Módulo de elasticidad (GPa) & 0,6 \\
\hline Densidad (Kg/m3) & 1,09 \\
\hline
\end{tabular}

\section{Manufactura del Biocompuesto}

Con la finalidad de elaborar los materiales biocompuestos se utilizó la técnica de infusión en vacío. Las diferentes capas de fibras de fique en configuración aleatoria se ubicaron en un molde horizontal de $350 \mathrm{~mm}$ por $350 \mathrm{~mm}$. En consecuencia, se aplicó el vacío mediante una bomba de vacío Vacuubrand 1C, a un 1 bar de presión, eliminando el exceso de aire atrapado. El curado se realizó a temperatura ambiente durante 24 horas. En la Figura 2 se observa la fabricación del biocompuesto.

Se obtuvo cuatro materiales biocompuestos con diferentes cantidades de capas de fique. En la Tabla 2 se describen cada uno de ellos.

Para cada uno de los materiales se fabricaron cinco probetas obtenidas de los laminados y cortadas mediante corte con chorro de agua, con las siguientes dimensiones $25,3 \pm 0,17 \mathrm{~mm}$ de ancho por $252 \pm 2,2 \mathrm{~mm}$ de largo por $2,52 \pm 0,08 \mathrm{~mm}$ de alto, según norma ASTM D3039/D3039M.

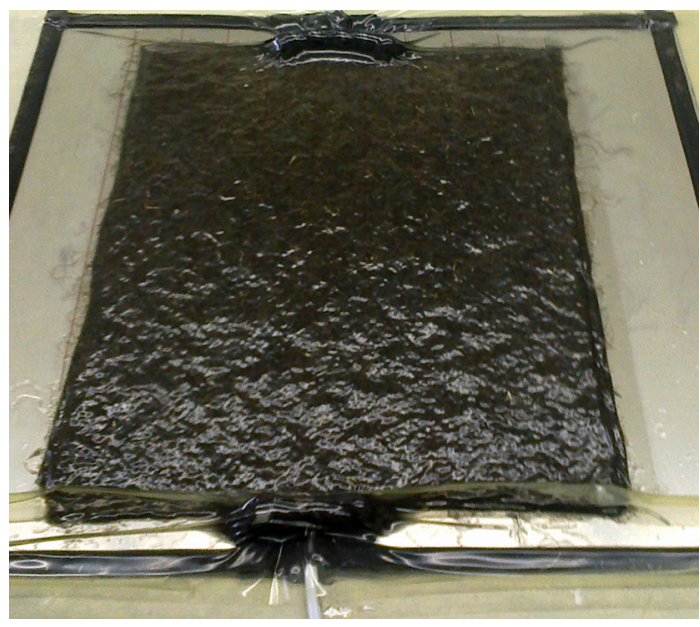

Figura 2. Montaje por infusión en vacío para la fabricación de los biocompuestos.

\section{Prueba dinámica Análisis Modal Experimental} El ensayo dinámico se llevó a cabo por medio de un análisis modal experimental realizando la medición de la respuesta en frecuencia (FRF) según metodología de la Norma ISO 7626-2 "mechanical vibration and shock-Experimental determination of mechanical mobility - Part 2: Measurements using single-point translation excitation with an attached vibration exciter".

El montaje de las probetas se realizó de forma libre (sin restricciones) usando elementos elásticos, ubicándose de forma fija en un shaker electrodinámico marca "The Modal Shop" (elemento de excitación), registrando la magnitud de la excitación por medio de un sensor de fuerza PCB 208C02 acoplado al extremo superior del equipo.

La amplitud de la deformación (debido a la vibración) en los biocompuestos se realizó con un acelerómetro PCB 352C68 en 5 puntos distribuidos uniformemente en la superficie total de las probetas, distanciados 5

Tabla 2. Biocompuestos de fibra de fique/resina de poliéster insaturado.

\begin{tabular}{|c|l|c|}
\hline Material & \multicolumn{1}{|c|}{ Descripción } & Densidad $\left(\mathbf{k g} / \mathbf{m}^{\mathbf{3}}\right)$ \\
\hline Biocompuesto 1 & Una capa de fique correspondiente a 5,26\% en peso & $1217,28 \pm 1,7$ \\
\hline Biocompuesto 2 & Dos capas de fique correspondiente al 10,45\% en peso & $1224,96 \pm 0,8$ \\
\hline Biocompuesto 3 & Tres capas de fique correspondiente al 15,36\% en peso & $1249,92 \pm 1,9$ \\
\hline Biocompuesto 4 & Cuatro capas de fique correspondiente al 20,09\% en peso & $1274,24 \pm 2,1$ \\
\hline
\end{tabular}


cm cada uno. En la Figura 3 se observa el montaje esquemático del ensayo.

Para cada una de las mediciones (Puntos), se realizaron un total de 5 pruebas, obteniendo el promedio de estas para cada material. El rango de excitación fue cubierto de 10 a $250 \mathrm{~Hz}$ usándose un barrido sinusoidal.

Las probetas a ensayar se unieron al sensor de fuerza utilizando pegante instantáneo en uno de sus extremos, así mismo el acelerómetro en cada uno de los puntos de medición se unió igualmente con pegante instantáneo. Adicionalmente, el extremo opuesto de la probeta se montó en elementos elásticos simulando condiciones de libertad como se muestra en la Figura 4.

Del ensayo de FRF se obtiene las gráficas de Magnitud y fase las cuales permiten identificar las frecuencias naturales y adicionalmente las del componente real e imaginario utilizadas para la obtención de la forma modal. (Ver Figura 5)

La extracción del amortiguamiento se realizó utilizándose el método Peak-picking siguiendo la ecuación (1).

$$
\xi=\frac{\Delta \omega}{2 \omega_{n}}
$$

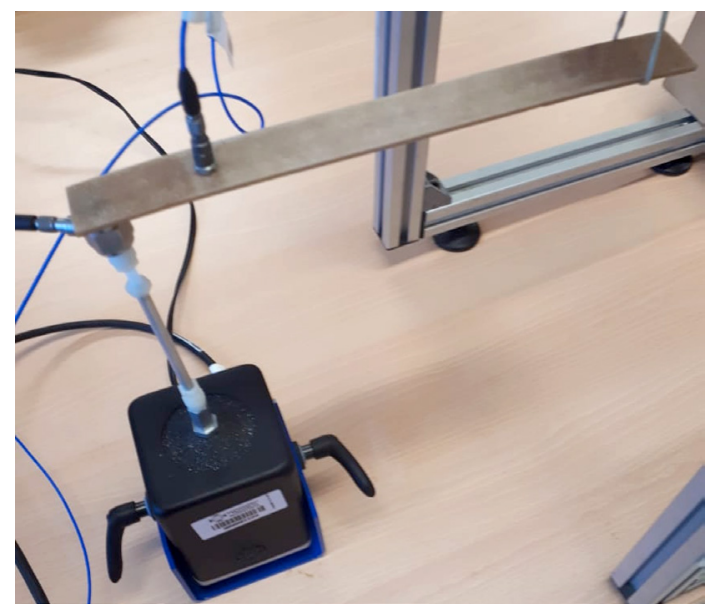

Figura 4. Montaje Probetas.

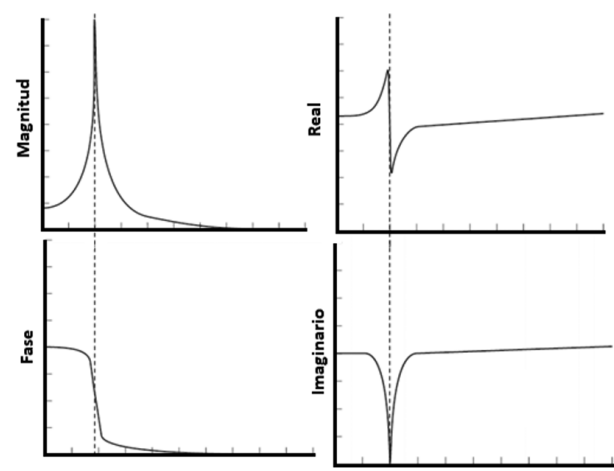

Figura 5. Graficas resultado del ensayo FRF.

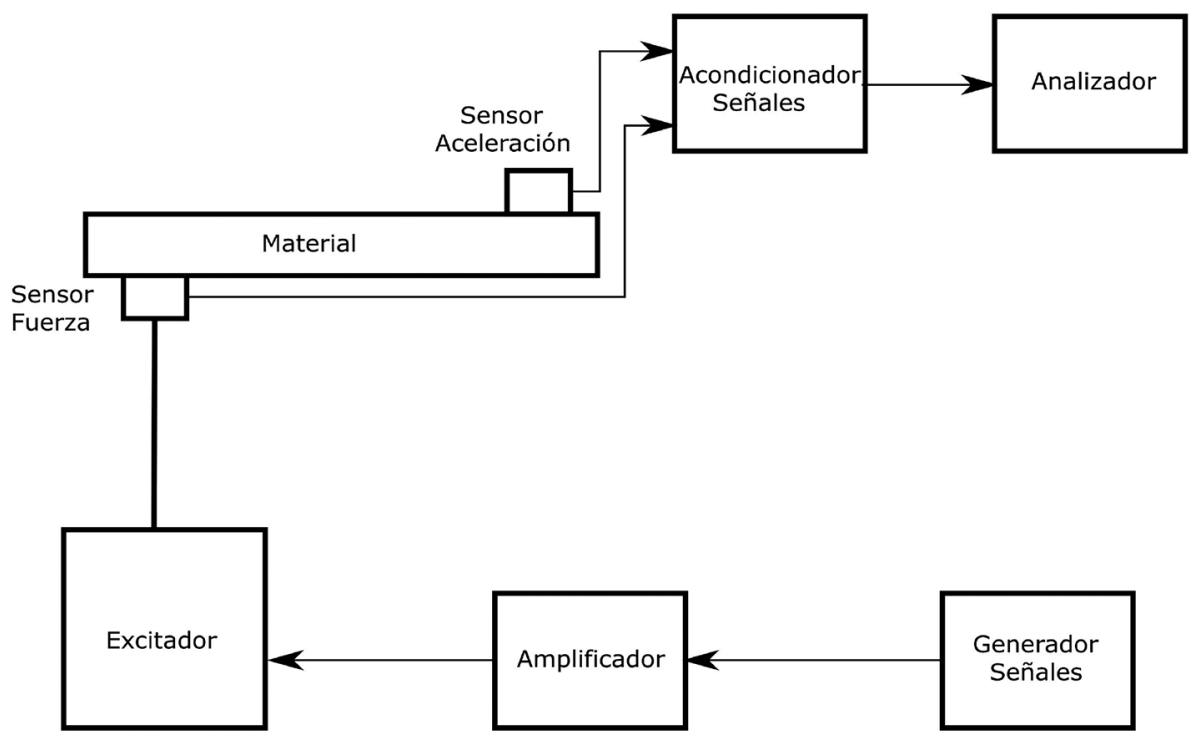

Figura 3. Montaje esquemático del Ensayo Prueba Dinámica. 
Donde $\xi$ es el amortiguamiento; $\Delta \omega$ es el ancho de banda y $\omega_{n} l$ la frecuencia natural. El valor de ancho de banda se determina por medio de la gráfica de magnitud obtenida del ensayo de FRF identificando los picos de las frecuencias naturales del cuerpo y obteniendo las frecuencias para las cuales la amplitud al cuadrado es la mitad del máximo valor de amplitud de la frecuencia natural, es decir una caída de $3 \mathrm{~dB}$ en el nivel con respecto al valor máximo [26] como se observa en la Figura 6.

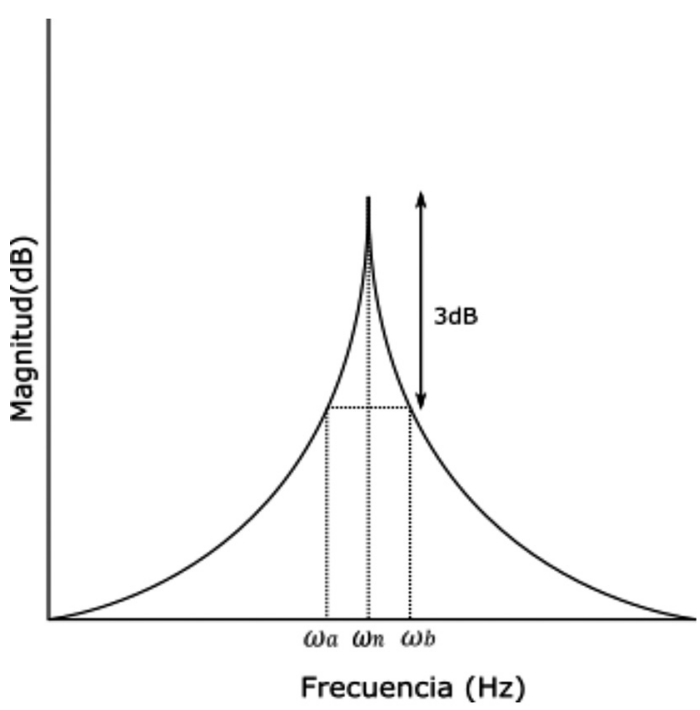

Figura 6. Método Peak-picking.

El ancho de banda se obtiene según la ecuación (2)

$$
\Delta \omega=\omega_{a}-\omega_{b}
$$

Para la obtención de la forma modal se utilizó la respuesta imaginaria del ensayo de FRF donde se unió las diferentes amplitudes de cada uno de los puntos de medición para conocer que patrón de deformación presentaron los biomateriales en cada uno de sus frecuencias naturales [27] como se observa en la Figura 7.

\section{RESULTADOS}

Los resultados obtenidos de los parámetros modales (Frecuencia natural, amortiguamiento y forma modal) de cada uno de los biocompuestos reforzados con fibra de fique se describen a continuación.

\section{Frecuencia Natural}

En el rango de 10 a $250 \mathrm{~Hz}$ se obtuvieron cuatro frecuencias naturales, (como se observa en la Tabla 3 ), para cada uno de los biocompuestos los cuales se denominan modos de vibración del material.

Según los resultados, se evidencia, que a medida que se incrementan las capas de fique, los valores de las frecuencias naturales tienden a aumentar, debido a que aumenta la rigidez del biocompuesto ya que se presentan anclajes de tipo mecánico en la interface fibra-matriz, que restringen la capacidad de deformación de la resina, primando la rigidez de la fibra lo que se opone a que el material se doble por la acción de fuerzas exteriores que estén actuando sobre su superficie, es decir la transmisión de la vibración generada por elemento excitador.

Este aumento también lo reporta Senthil et al. [24] en biocompuesto de poliéster-banana en el cual al

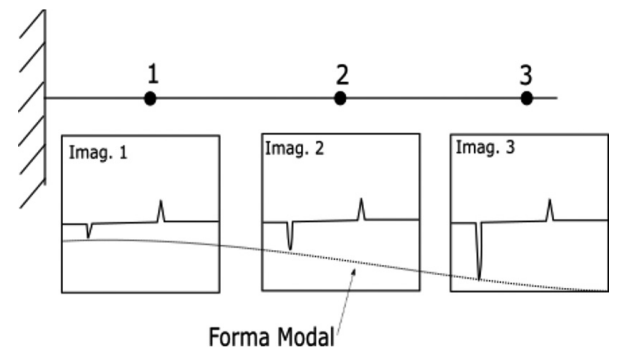

Figura 7. Método Peak-picking.

Tabla 3. Frecuencia natural de los materiales obtenidos.

\begin{tabular}{|c|c|c|c|c|c|}
\hline \multirow{2}{*}{$\begin{array}{c}\text { Modo de } \\
\text { vibración }\end{array}$} & \multicolumn{5}{|c|}{ Frecuencia natural (Hz) } \\
\cline { 2 - 6 } & Poliéster & Biocompuesto 1 & Biocompuesto 2 & Biocompuesto 3 & Biocompuesto 4 \\
\hline 1 & $43,75 \pm 2,8$ & $44,5 \pm 4,2$ & $48,75 \pm 2,8$ & $50 \pm 4,4$ & $57,5 \pm 1,2$ \\
\hline 2 & $112,5 \pm 3,6$ & $113,75 \pm 3,0$ & $118,5 \pm 1,8$ & $119,25 \pm 3,2$ & $123,5 \pm 4,1$ \\
\hline 3 & $196,25 \pm 1,9$ & $197,5 \pm 1,2$ & $202,5 \pm 5,1$ & $204,75 \pm 7,8$ & $209,75 \pm 6,4$ \\
\hline 4 & $217,75 \pm 4,6$ & $219,25 \pm 5,2$ & $222,5 \pm 6,4$ & $225,75 \pm 8,2$ & $232,5 \pm 7,1$ \\
\hline
\end{tabular}


$30 \%$ en peso de fibra obtuvo, en su segundo modo de vibración, una frecuencia natural de $177,6 \mathrm{~Hz}$ mientras que con $40 \%$ una frecuencia de $183,1 \mathrm{~Hz}$ y con $50 \%$ una de $189,8 \mathrm{~Hz}$. Igualmente Rajesh et al. [28] en biocompuesto de poliéster con una capa tejida de banana reporto una frecuencia natural de $42 \mathrm{~Hz}$ en su primer modo de vibración mientras que con dos capas aumento a $59 \mathrm{~Hz}$.

Es Importante resaltar, que con el incremento de cantidad de las fibras naturales en los biocompuestos se aumenta la densidad, la cual, disminuye la frecuencia natural, debido al incremento de la inercia que debe vencer el material para vibrar. Es así, que en los biocompuestos con fibra de fique en su frecuencia natural el aumento de la rigidez fue más significativo, que el de la masa.

Las dimensiones (especialmente el espesor) influyen directamente en las frecuencias naturales de los biocompuestos, sin embargo, para la comparación de los resultados de los diferentes materiales fabricados su afectación no se toma como significativa, debido, a que las geometrías de las probetas eran las mismas.

La resonancia mecánica es un fenómeno que se produce cuando un cuerpo por la acción de una fuerza externa vibra a la misma periodicidad que la frecuencia natural del mismo produciendo un aumento en la amplitud el cual puedes ser destructivo [29]. En el caso de los materiales compuestos producen efectos de delaminación como lo reporta Mukhopadhyaya et al. [30], es por esta razón que es importante conocer los parámetros que puedan modificar las frecuencias naturales, como el aumento de cantidad de fibra natural, evitando de esta forma daños por vibración en aplicaciones de tipo industrial.

Durante la manufactura de los biomateriales se detectaron porosidades debido a que burbujas de aire quedaban atrapadas cuando se realizaba la mezcla resina catalizador y en el momento de succión cuando ingresaba al sistema de vacío. Este fenómeno pudo afectar los resultados sin embargo se aminoró aumentando el tiempo de pre-impregnado lo que se reflejó en mayor cantidad de resina utilizada. Esta anomalía es característica del sistema de manufactura infusión en vacío y se reporta en trabajos similares de materiales compuestos [3134]. Por tal razón, y con miras a disminuir estas imperfecciones, se recomienda para futuros trabajos implementar técnicas de mitigación en el momento de la fabricación como las que indica Lee Hamill et al. [35] en su investigación.

\section{Amortiguamiento}

En la Figura 8, se muestran los valores para el amortiguamiento de la resina pura y los compuestos con fibra de fique en los diferentes modos de vibración, donde se observan altos valores debido al comportamiento viscoelástico de la resina y un pequeño aumento con el número de capas de fibra natural incorporado en los biocompuestos.

Este aumento se debe al comportamiento de la interfaz de los compuestos, debido a que al existir

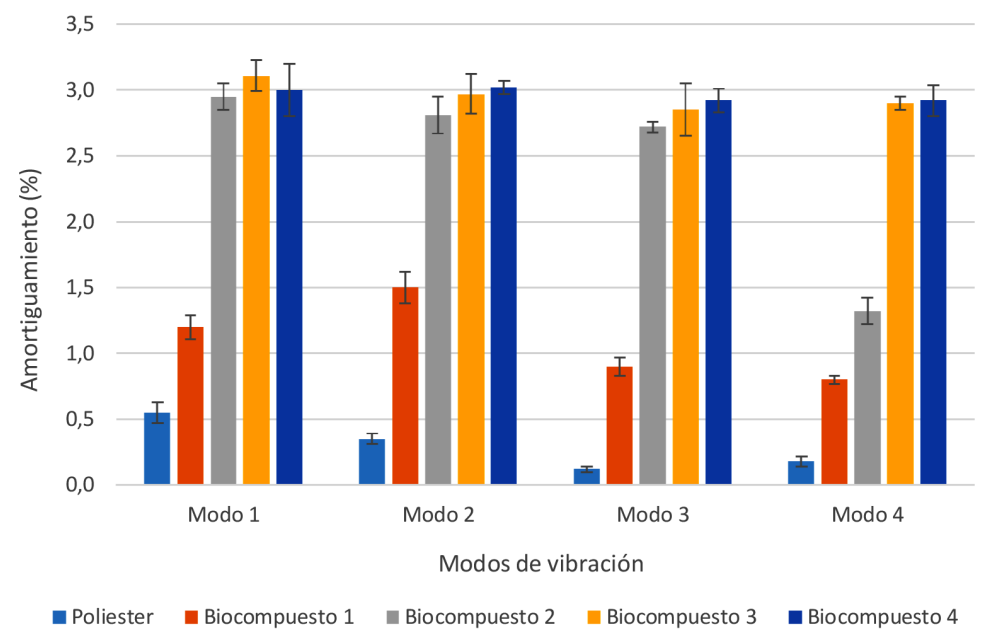

Figura 8. Amortiguamiento de los compuestos ensayados. 
más cantidad de fibra hay mayores deslizamientos en las regiones no unidas entre la fibra y matriz produciendo delaminaciones y movimientos internos donde se disipa la energía como lo describe $\mathrm{Li}$ Ma et al. [36] en compuesto de fibra de carbono, adicionalmente hay una mayor cantidad espacios de aire, por la porosidad de la fibra, que permiten un aumento en la transferencia de calor, lo que conlleva a que la energía se transforme en calor.

No existe un patrón uniforme en las tendencias relacionados con los modos de vibración y la cantidad de fibra debido a que no solo el contenido de la fibra influye en el amortiguamiento, adicionalmente la rigidez, el espesor de la interfaz afectan esta propiedad del material, para la cual el proceso de manufactura desempeña un papel fundamental, especialmente la presión de vacío como lo menciona Chandra et al. en review de estudio de amortiguamiento de materiales fibro reforzados [37]. Adicionalmente Lee et al. [38] atribuyen estas dispersiones a la anisotropía del material.

Los valores de amortiguamiento obtenidos en los biomateriales de fique, presentaron una magnitud similar a los reportados por Loan et al. [21] en compuestos de fibra de vidrio utilizando una matriz de poliéster como la usada en del presente estudio.

La magnitud del amortiguamiento presentado por los biocompuestos indican que permiten disipar alta energía por vibraciones o impactos, lo que hace posible emplearlos en aplicaciones donde se requieran aisladores de vibración o sonido como lo sugieren Akash et al. [39] y Genc et al. [40] en investigaciones dinámicas realizadas a biocompuestos con otro tipo de fibras naturales.

Para la extracción de parámetros modales en posteriores estudios de investigación se recomienda aplicar metodologías más robustas que el Peakpicking como lo es el Circle-Fitting o Least-squares como lo describen He et al. [41].

\section{Forma Modal}

La forma modal o forma en que se deformó el eje en cada una de sus modos de vibración (Frecuencias naturales) se obtuvo de las gráficas del componente imaginario resultado del análisis de FRF siendo ellas las que se observan en la Figura 9.

Se observa que, los cuatro modos de vibración de los biocompuestos se obtuvieron de forma flectora, esto muy posiblemente se atribuye a que la probeta es mucho más larga que ancha permitiendo que se deforme más fácilmente en esta dirección y no torsionalmente.

Así mismo, la forma modal por modo de cada uno de los biocompuestos es similar a causa de la misma geometría en las probetas.

Hay que tener en cuenta que estas graficas son una aproximación a la forma de deformación de los

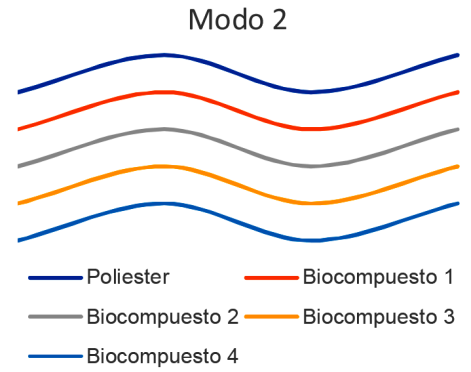

Modo 4
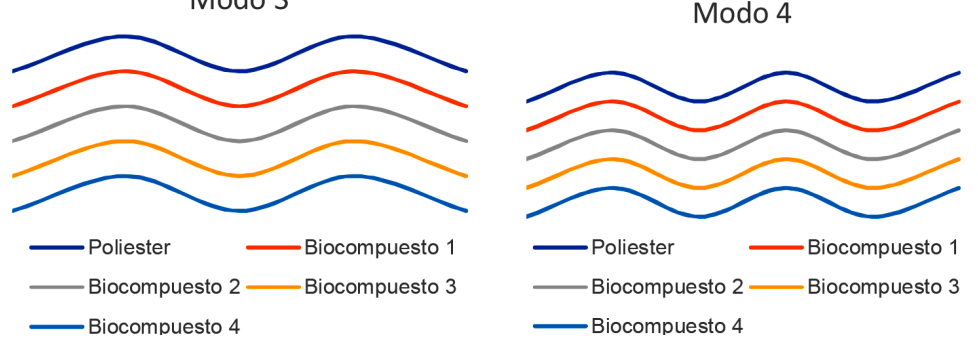

Figura 9. Forma Modal por Modo de los materiales compuestos ensayados. 
biocompuestos en cada uno de sus modos de vibración y que los mismos pueden variar dependiendo de la amplitud de la fuerza que se le aplique como excitación a los biomateriales.

Para estudios más concluyentes de la aplicación de estos biomateriales en aplicaciones dinámicas, se debe evaluar también los diferentes modos torsionales.

\section{CONCLUSIONES}

Se evidenció que las frecuencias naturales de los biocompuestos con más cantidad de fibra natural, son más altas debido a que el material aumenta su rigidez la cual genera más oposición a la deformación del material por la transmisión de la vibración evitando de esta forma que se deflecte. Es por esta razón que se presenta en el primer modo de vibración un aumento del $1,7 \%$ en la frecuencia natural del material de solo poliéster al material biocompuesto de una capa de fique $(5,26 \%$ en peso), mientras que para el material biocompuesto con dos capas de fique $(10,45 \%$ en peso) fue del 11,4\%; adicionalmente él material biocompuesto con tres capas de fique $(15,36 \%$ en peso) presento un incremento del $14,3 \%$ y por último se obtuvo un aumento máximo de 31,4\% en material biocompuesto con cuatro capas de fique $(20,09 \%$ en peso). Este comportamiento de crecimiento se evidencio adicionalmente en el segundo, tercer y cuarto modo de vibración de los biocompuestos.

El amortiguamiento presento una alta magnitud, lo que hace posible emplearlos en aplicaciones como aisladores de vibración o sonido, así como un aumento con la inserción de la fibra natural de fique, es así como en el primer modo de vibración se aumenta de $0,4 \%$ de amortiguamiento en el material de solo poliéster a $1,2 \%$ con una capa de fibra natural, $3,0 \%$ con dos capas de fibra natural $3,1 \%$ con tres capas de fibra y $3,0 \%$ con cuatro capas. Este aumento se debió en parte a la alta porosidad de las fibras y a la falta de adherencia entre la fibra y la matriz que genera espacios permitiendo que la energía se disipe con mayor facilidad, sin embargo, el comportamiento de crecimiento no fue lineal ya que es afectado por otros factores como el espesor de la interfaz los cuales dependen directamente del sistema de manufactura.

Se obtuvo una forma modal en flexión para cada uno de los modos de vibración de los biocompuestos aumentando el número de nodos cada vez que aumenta el valor de la frecuencia natural debido a la geometría de la probeta y el rango de excitación de la misma.

\section{AGRADECIMIENTOS}

Agradecimiento especial al laboratorio de vibraciones de la Universidad Pontificia Bolivariana seccional Bucaramanga quien proporcionó los equipos para la ejecución de la de medición de la respuesta en frecuencia y al laboratorio de materiales de la Universidad de Pamplona quien fabricó las probetas del material compuesto reforzado con fibras de fique ensayadas.

\section{REFERENCIAS}

[1] M.A. Hidalgo-salazar and J.P. Correa. "Results in Physics Mechanical and thermal properties of biocomposites from nonwoven industrial Fique fiber mats with Epoxy Resin and Linear Low Density Polyethylene". Results Phys. Vol. 8, pp. 461-467. 2018.

[2] P. Gañán and I. Mondragon. "Fique fiberreinforced polyester composites: Effects of fiber surface treatments on mechanical behavior". J. Mater. Sci. Vol. 39, pp. 31213128. 2004.

[3] P. Gañán and I. Mondragon. "Surface modification of fique fibers. Effect on their physico-mechanical properties". Polym. Compos. Vol. 23 No 3, pp. 383-394. 2002.

[4] M.A. Hidalgo, M.F. Muñoz, and K.J. Quintana. "Desempeño mecánico del compuesto polietileno-aluminio reforzado con agro fibras continuas de fique". Rev. Lat. Met. Mat. Vol. 31 N$^{\circ}$ 2, pp. 187-194. 2011.

[5] V. Balaji, K. Lau, D. Hui, and D. Bhattacharyya. "Graphene-based materials and their composites: A review on production, applications and product limitations". Compos. Part B, Vol. 142, pp. 200-220. January 2018.

[6] H. Materials, L.L. Dong, M. Ahangarkani, W.G. Chen, and Y.S. Zhang. "Recent progress in development of tungsten-copper composites: Fabrication, modification and applications". Int. J. Refract. Metals Hard Mater. Vol. 75, pp. 30-42. February 2018.

[7] D.B. Dittenber and H.V.S. Gangarao. "Critical review of recent publications on 
use of natural composites in infrastructure". Compos. Part A Appl. Sci. Manuf. Vol. 43 No 8, pp. 1419-1429. 2012.

[8] P. Luna, A. Mariño, J. Lizarazo-marriaga, and O. Beltrán. "Dry etching plasma applied to fique fibers: influence on their mechanical properties and surface appearance". Procedia Eng. Vol. 200, pp. 141-147. 2017.

[9] X. Li, L. G. Tabil, and S. Panigrahi. "Chemical Treatments of Natural Fiber for Use in Natural Fiber-Reinforced Composites: A Review". J. Polym. Environ. Vol. $15 \mathrm{~N}^{\circ}$ 1, pp. 25-33. 2007.

[10] M.A. Hidalgo, M. Muñoz, and K. Quintana. "Análisis Mecánico del compuesto polietileno aluminio reforzado con fibras cortas de fique en disposición bidimensional". Rev. Lat. Met., Vol. 32 No 1, pp. 89-95. 2012.

[11] M.A. Hidalgo, M.F. Muñoz, and J.H. Mina. "Influence of Incorporation of Natural Fibers on the Physical, Mechanical, and Thermal Properties of Composites LDPE-Al Reinforced with Fique Fibers". Int. J. Polym. Sci. Vol. 2015. Article ID 386325. 2015.

[12] J.H. Mina. "Caracterización Físico-Mecánica De Un Almidón Termoplastico De Yuca Y Análisis Interfacial Con Fibras De Fique". Biotecnol. en el Sect. Agropecu. y Agroindustrial. Vol. $10 \mathrm{~N}^{\circ}$ 2, pp. 99-110. 2012.

[13] D.P. Navia, A.A. Ayala, and H.S. Villada. "Effect of cassava flour gelatinization on mechanical properties of bioplastics". Biotecnol. en el Sect. Agropecu. y Agroindustrial. Vol. $13 \mathrm{~N}^{\circ}$ 1, pp. 38-44. 2015.

[14] S.D. Arjona, F. Perdomo, and R.M. De Gutiérrez. "Ecolaminados de pead-fibras de fique". Ing. y Compet. Vol. 3 N$^{\circ}$ 1, pp. 43-50. 2001.

[15] C. Gómez Hoyos and A. Vázquez. "Flexural properties loss of unidirectional epoxy/fique composites immersed in water and alkaline medium for construction application". Compos. Part B Eng. Vol. 43 No 8, pp. 31203130. 2012.

[16] M.A. Hidalgo-Salazar, J.H. Mina, and P.J. Herrera-Franco. "The effect of interfacial adhesion on the creep behaviour of LDPEAl-Fique composite materials". Compos. Part B Eng. Vol. 55, pp. 345-351. 2013.

[17] P. Gañán and I. Mondragon. "Thermal and degradation behavior of fique fiber reinforced thermoplastic matrix composites". J. Therm. Anal. Calorim. Vol. 73 No3, pp. 783-795. 2003.

[18] P. Gañán and I. Mondragon. "Influence of Compatibilization Treatments on the Mechanical Properties of Fique Fiber Reinforced Polypropylene Composites". Int. J. Polym. Mater. Vol. 53 No 11, pp. 997-1013. 2004.

[19] F. Klimenda and J. Soukup. "Modal Analysis of Thin Aluminium Plate". Procedia Eng. Vol. 177, pp. 11-16. 2017.

[20] É.L. Oliveira, N.M.M. Maia, A.G. Marto, R.G.A. da Silva, F.J. Afonso, and A. Suleman. "Modal characterization of composite flat plate models using piezoelectric transducers". Mech. Syst. Signal Process. Vol. 79, pp. 16-29. 2016.

[21] V.C. Ioan Curtu, M.D. Stanciu. "The Modal Analysis of Plates Made of Woven Composite Materials". Bul. AGIR nr. Vol. $8204 \mathrm{~N}^{\circ} 1$, pp. 2-5. 2011.

[22] C. Garcia, J. Wilson, I. Trendafilova, and L. Yang. "Vibratory behaviour of glass fibre reinforced polymer (GFRP) interleaved with nylon nanofibers". Compos. Struct. Vol. 176, pp. 923-932. 2017.

[23] K. Wang, K. Okuno, M. Banu, and B.I. Epureanu. "Vibration-based identification of interphase properties in long fiber reinforced composites". Compos. Struct. Vol. 174, pp. 244-251. 2017.

[24] K. Senthil Kumar, I. Siva, P. Jeyaraj, J.T. Winowlin Jappes, S.C. Amico, and N. Rajini. "Synergy of fiber length and content on free vibration and damping behavior of natural fiber reinforced polyester composite beams". Mater. Des. Vol. 56, pp. 379-386. October 2014.

[25] S. Gómez, B. Ramón, and R. Guzman. "Comparative study of the mechanical and vibratory properties of a composite reinforced with fique fibers versus a composite with E-glass fibers". Rev. UIS Ing. Vol. $17 \mathrm{~N}^{\circ} 1$, pp. 43-50. 2018.

[26] N. Rajini, J.T.W. Jappes, S. Rajakarunakaran, and P. Jeyaraj. "Mechanical and free vibration properties of montmorillonite clay dispersed with naturally woven coconut sheath composite". J. Reinf. Plast. Compos. Vol. 31 $\mathrm{N}^{\circ}$ 20, pp. 1364-1376. 2012.

[27] J. He and Z.-F. Fu. "7 - Frequency response function measurement". In Modal Analysis, 
J. He and Z.-F. Fu, Eds. Oxford: ButterworthHeinemann, pp. 140-158. 2001.

[28] M. Rajesh and J. Pitchaimani. "Dynamic mechanical analysis and free vibration behavior of intra-ply woven natural fiber hybrid polymer composite". J. Reinf. Plast. Compos. Vol. 35, № 3, pp. 228-242. 2016.

[29] A. Peralta, P. Reyes, and A. God. "El fenómeno de la resonancia". Lat. Am. J. Phys. Educ. Vol. $3 \mathrm{~N}^{\circ}$ 3, pp. 612-618. 2009.

[30] T. Mukhopadhyay, S. Naskar, P.K. Karsh, S. Dey, and Z. You. "Effect of delamination on the stochastic natural frequencies of composite laminates". Compos. Part B Eng. Vol. $154 \mathrm{~N}^{\circ}$ March, pp. 242-256. 2018.

[31] D.C. Darrow, P.A. Propatic, and T.H. Brayden, Jr. "Elimination of mold surface porosity on composite parts". J. Adv. Mater. Vol. 27, pp. 41-46. 1995.

[32] L. Hamill, T. Centea, G. Nilakantan, and S. Nutt. "Surface Porosity in Out-of-Autoclave Prepreg Processing: Causes and Reduction Strategies". International SAMPE Technical Conference. 2014.

[33] M.L. Herring, J.I. Mardel, and B.L. Fox. "The effect of material selection and manufacturing process on the surface finish of carbon fibre composites". J. Mater. Process. Technol. Vol. $210 \mathrm{~N}^{\circ}$ 6-7, pp. 926-940. 2010.

[34] M.L. Herring and B.L. Fox. "The effect of a rapid curing process on the surface finish of a carbon fibre epoxy composite". Compos. Part B Eng. Vol. 42 N 5, pp. 1035-1043. 2011.
[35] L. Hamill, T. Centea, and S. Nutt. "Surface porosity during vacuum bag-only prepreg processing: Causes and mitigation strategies". Compos. Part A Appl. Sci. Manuf. Vol. 75, pp. 1-10. 2015.

[36] L. Ma et al. "Modal characteristics and damping enhancement of carbon fiber composite auxetic double-arrow corrugated sandwich panels". Compos. Struct. Vol. 203, pp. 539-550. May 2018.

[37] R. Chandra, S.P. Singh, and K. Gupta. "Damping studies in fiber-reinforced composites - a review". Compos. Struct. Vol. $46 \mathrm{~N}^{\circ}$ 1, pp. 41-51. 1999.

[38] C.R. Lee and T.Y. Kam. "Identification of mechanical properties of elastically restrained laminated composite plates using vibration data". J. Sound Vib. Vol. 295 Nº 3-5, pp. 9991016. 2006.

[39] D.A. Akash, N. Thyagaraj, and L. Sudev. "Experimental study of dynamic behaviour of hybrid jute/sisal fibre reinforced polyester composites". Int. J. Sci. Eng. Appl. Vol. 2 $\mathrm{N}^{\circ}$ 8, pp. 170-172. 2013.

[40] G. Genc and H. Koruk. "Identification of the Dynamic Characteristics of Luffa Fiber Reinforced Bio-Composite Plates". Bioresources. Vol. 12, pp. 5358-5368. 2017.

[41] J. He and Z.-F. Fu. "8 - Modal analysis methods - frequency domain". In Modal Analysis, J. He and Z.-F. Fu, Eds. Oxford: Butterworth-Heinemann, pp. 159-179. 2001. 